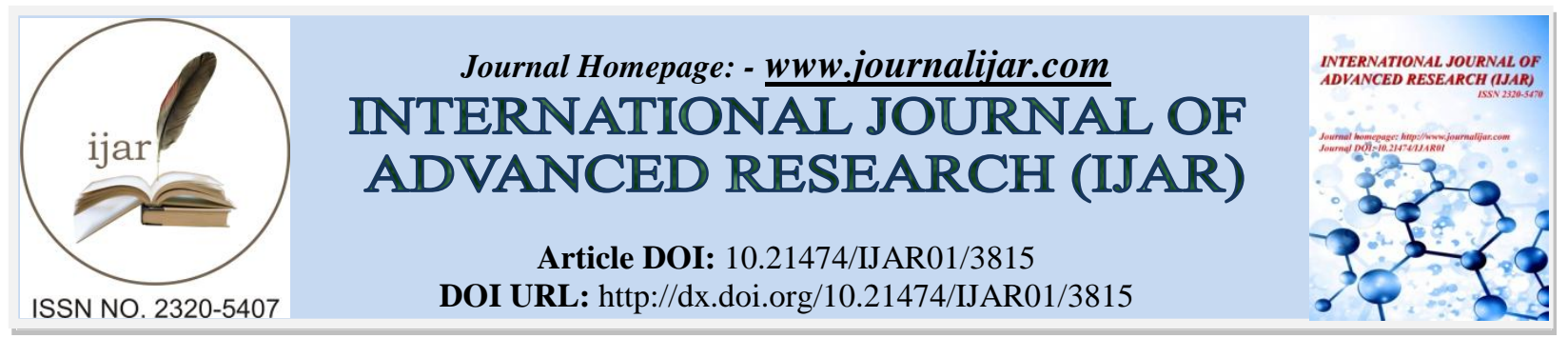

RESEARCH ARTICLE

\title{
HEMODYNAMIC INDEXES, REGISTERED IN YOUNG SUBJECTS WITH HIGH NORMAL BLOOD PRESSURE.
}

\author{
${ }^{*}$ Mariana Bacelova ${ }^{1}$, Julia Nikolova ${ }^{1}$, Peter Nikolov ${ }^{2}$, Maria Bacelova ${ }^{3}$ and Maria Negreva ${ }^{4}$. \\ 1. Department of Physiology, Medical Faculty, Medical University-Plovdiv. \\ 2. First department of internal diseases - cardiology section, Medical Faculty, Medical University - Plovdiv. \\ 3. Student $2^{\text {nd }}$ course dental medicine, Dental faculty, Medical University, Plovdiv. \\ 4. Department of internal diseases - cardiology section, Medical Faculty, Medical University - Varna.
}

\section{Manuscript Info}

[.........................

Manuscript History

Received: 07 February 2017

Final Accepted: 02 March 2017

Published: April 2017

\section{Key words:-}

hemodynamic indices; high normal arterial pressure; microcirculation.

\section{Abstract}

The goal of the study was to examine microcirculation and the hemodynamic profile in individuals with high normal arterial pressure (HNAP). The object of the investigation is focused on young individuals from 18 to 35 years with HNAP. The two following groups - with HNAP and with optimal normal arterial pressure (ONBP) are formed, based on inquiry and screening arterial pressure measurement among 109 individuals (49 men and 60 women). Non invasive native capillaroscopy and anthropometric examination, arterial pressure monitoring and non invasive bio impedance cardiography were carried out.

Results: Significantly higher crossed capillaries percentage (22.34 $\pm 13.07, \mathrm{p}<0.05)$ is registered in HNAP group compared to the controls - 16.36 \pm 16.64 . Cardiac output /cardiac index ratio and velocity index were increased in HNAP, as well as mean arterial pressure. Conclusion: hemodynamic evaluations in HNAP group are close to these in hypertensive individuals.

Copy Right, IJAR, 2017,. All rights reserved.

\section{Introduction:}

Elevated arterial blood pressure (ABP) is an important cardiovascular risk factor for cardiovascular morbidity and mortality worldwide [1]. The European Society of Cardiology (ESC) defines ABP values under 140/90 $\mathrm{mmHg}$ in three categories - optimal (under 120/80 $\mathrm{mmHg}$ ), normal $(120-129 \mathrm{mmHg}$ for systolic and/or $80-84 \mathrm{mmHg}$ for diastolic) and high normal (130-139 $\mathrm{mmHg}$ for systolic and/or 85-89 $\mathrm{mmHg}$ for diastolic) in concern with the risk of cardiovascular disease or stroke to appear following arterial blood pressure abnormalities [2,3].The risk, arterial hypertension (AH) to develop is higher in individuals with high normal ABP (HNAP) [4,5,6,7,8 ], as it is considered to be characterised by endothelial activation and metabolic changes $[9,10]$. Native capillaroscopy is a non invasive method allowing direct visualization of the nutritive dermal blood vessels in vivo. Bioimpedance cardiography (BIC) is based on electrical voltage changes registration in the thoracic region, giving information about the hemodynamics $[16,17,18,19]$.

\section{Aim:}

The goal of the study was to examine microcirculation and to follow the hemodynamic profile in young HNAP individuals with non invasive techniques. 


\section{Methods:}

After measuring ABP, recorded four times at 3 min intervals using Argus LCM plus, Shiller device, among 109 individuals at age of $20 \pm 1.3$ years ( 49 men and 60 women), two groups were formed: HNAP, $n=50$ and control with optimal ABP (ONAP), $n=59$.

The protocol of the study for both of the groups was:

1. Native capillaroscopy (Capillaroscope JH1005, PRC), magnification $\times 250$;

2. Anthropometric examination with Guangzhou ZYS Electric CO, Ltd, PRC;

3. Five minutes recording of ABP by CNAP "HD" (Biopac Instruments, USA) $[13,14,15]$;

4. Five minutes bioimpedance cardiography by NICO-100C (Biopac Instruments, USA).

The study was approved by the Medical University- Plovdiv's medical ethics committee (protocol 4/16.06, 2016).

\section{The followed up parameters were:}

Mean arterial / venous capillary diameter $(\mathrm{a} / \mathrm{v})$;

Number of capillaries;

Crossed capillaries, $(\%)$;

Abnormal capillaries, (\%);

Body Mass Index $\left(\mathrm{BMI}=\mathrm{kg} / \mathrm{m}^{2}\right)[11,12]$;

Visceral fat index $\left(\mathrm{VFI} \mathrm{kg} / \mathrm{m}^{2}\right)$;

Basal metabolic rate (BMR Kcal/d);

Fat free mass index (FFMI kg/m²);

Metabolic age (years).

The hemodynamic indices, followed up by BIC ,CNAP and capillaroscopic parameters are presented in details in table 2 .

The data were statistically processed by SPSS 16 package - descriptive analysis and paired t-test was used.

\section{Results:}

Significantly higher crossed capillaries percentage $(22.34 \pm 13.07, \mathrm{p}<0.05)$ is registered in HNAP group compared to the control one $-(16.36 \pm 16.64)$, fig. 1 .

Significantly higher are BMI $(23.45 \pm 4.25 \mathrm{p}<0.01)$, VFI $(6.73 \pm 5.51 \mathrm{p}<0.01)$, FFMI $(18.04 \pm 2.19 \mathrm{p}<0.01)$, BMR $(1731.94 \pm 344.59 \mathrm{p}<0.01)$ and Metabolic age $(22.14 \pm 6.24 \mathrm{p}<0.01)$ in HNAP compared to the controls BMI (20.83 \pm 5.17$)$, VFI $(2.89 \pm 1.83)$, FFMI (15.75 \pm 1.38$)$, BMR $(1435.51 \pm 255.84)$ and metabolic age (20.42 \pm 3.95$)$ (table 1). The MBP was higher $(96.36 \pm 9.09 \mathrm{p}<0.05)$ compared to the controls $(83.43 \pm 6.87)$.The mean values of velocity index ,VI $(0.05 \pm 0.1, \mathrm{p}<0.05)$ and cardiac output/cardiac index ratio, $\mathrm{CO} / \mathrm{CI}(1.86 \pm 0.26, \mathrm{p}<0.05)$ are significantly higher in HNAP in comparison to ONAP group, $(0.04 \pm 0.01),(1.65 \pm 0.17)$ respectively. Vascular resistance shows tendency of increasing in HNAP individuals, compared to ONAP group (table2).

\section{Discussion:}

We do not find vascular capillary remodeling ( $\mathrm{a} / \mathrm{v}$ changes) and capillary rarefaction in HNAB group, followed by us (table 2), as it is commented in the literature [20, 21, 23]. Increased crossed capillaries [22] and abnormal capillaries are registered in HNAP. Our results confirm the importance of overweight on the risk arterial hypertention to develop. In this regard, the risk profile correction in HNAP objects will prevent the AH appearance $[24,25,26]$. In HNAP were increased CO/CI ratio and VI, as well was MBP [27].

\section{Conclusion:}

The microcirculatory changes in young individuals with HNAP show prevailing morphological changes.

The hemodynamic evaluation in HNAP is close to that in the hypertensive individuals as it is commented in the literature[28]. The recommendations would be directed to changes in lifestyle - risk profile correction and increased physical activity would delay AH appearance. 
Table 1: Anthropometric analysis in ONAP and HNAP groups.

\begin{tabular}{|c|c|c|c|}
\hline $\begin{array}{c}\text { Groups } \\
\text { Parameters }\end{array}$ & $\begin{array}{c}\text { ONAP } \\
\text { Mean } \pm \text { SD } \\
\mathbf{N}=\mathbf{5 0}\end{array}$ & $\begin{array}{c}\text { HNAP } \\
\text { Mean } \pm \text { SD } \\
N=44\end{array}$ & $\mathbf{P}$ \\
\hline $\begin{array}{c}\text { BMI } \\
\left(\mathrm{kg} / \mathrm{m}^{2}\right)\end{array}$ & $20.83 \pm 5.17$ & $23.45 \pm 4.25$ & $<0.01 * *$ \\
\hline $\begin{array}{c}\text { VFI } \\
\left(\mathrm{kg} / \mathrm{m}^{2}\right)\end{array}$ & $2.89 \pm 1.83$ & $6.73 \pm 5.51$ & $<0.01 * *$ \\
\hline $\begin{array}{c}\text { FFMI } \\
\left(\mathrm{kg} / \mathrm{m}^{2}\right) \\
\end{array}$ & $15.75 \pm 1.38$ & $18.04 \pm 2.19$ & $<0.01 *$ \\
\hline $\begin{array}{c}\text { BMR } \\
(\text { Kcal/d })\end{array}$ & $1435.51 \pm 255.84$ & $1731.94 \pm 344.59$ & $<0.01 *$ \\
\hline Metabolic age ( years ) & $20.42 \pm 3.95$ & $22.14 \pm 6.24$ & $<0.01 * *$ \\
\hline
\end{tabular}

*Total examinated-94, men -49, women-45; ONAP-optimal normal arterial pressure; HNAP-high normal arterial pressure.

BMI-body mass index; VFI-visceral fat index; FFMI-fat free mass index; BMR-basal metabolic rate; metabolic age.

Table 2: Hemodynamic indices in ONAP and HNAP groups:

\begin{tabular}{|c|c|c|c|}
\hline $\begin{array}{l}\text { GROUPS } \\
\text { INDICES }\end{array}$ & $\begin{array}{l}\text { ONAP } \\
\text { Mean } \pm \text { SD } \\
\text { N=35 }\end{array}$ & $\begin{array}{l}\text { HNAP } \\
\text { Mean } \pm \text { SD } \\
\text { N=33 }\end{array}$ & $\mathbf{P}$ \\
\hline HR (imp /min ) & $86.79 \pm 13.27$ & $86.07 \pm 12.82$ & $>0.05$ \\
\hline 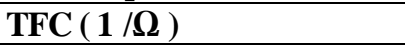 & $0.04 \pm 0.042$ & $0.04 \pm 0.01$ & $>0.05$ \\
\hline VI $(1 / \mathbf{s})$ & $0.04 \pm 0.01$ & $0.05 \pm 0.1$ & $<0.05 *$ \\
\hline SV ( ml / beat ) & $75.61 \pm 24.03$ & $84.49 \pm 28.43$ & $>0.05$ \\
\hline SV / SI & $1.65 \pm 0.17$ & $1.86 \pm 0.26$ & $<0.05 *$ \\
\hline CO (1/min) & $6.36 \pm 1.76$ & $7.22 \pm 2.11$ & $>0.05$ \\
\hline $\mathrm{CI}(\mathrm{L} / \mathrm{min}) / \mathrm{m}^{2}$ & $3.86 \pm 1.030$ & $3.87 \pm 0.94$ & $>0.05$ \\
\hline $\mathrm{CO} / \mathrm{CI}$ & $1.65 \pm 0.17$ & $1.86 \pm 0.26$ & $<0.05 *$ \\
\hline SVRI (dynes.S.cm $\left.{ }^{2} . \mathrm{cm}^{-5}\right)$ & $1778.49 \pm 619.55$ & $1929.37 \pm 635.24$ & $>0.05$ \\
\hline LCWI ( gm.m/m²) & $6.08 \pm 1.78$ & $6.70 \pm 1.96$ & $>0.05$ \\
\hline STR & $0.44 \pm 0.07$ & $0.46 \pm 0.11$ & $>0.05$ \\
\hline MBP ( mm Hg ) & $83.43 \pm 6.87$ & $96.36 \pm 9.09$ & $<0.05 *$ \\
\hline Number of capillaries & $9.17 \pm 2.261$ & $9.82 \pm 2.72$ & $>0.05$ \\
\hline Abnormal capillaries, (\%) & $12,50 \pm 11,68$ & $16,36 \pm 16,64$ & $>0.05$ \\
\hline Crossed capillaries (\%) & $16.36 \pm 16.64$ & $22.34 \pm 13.07$ & $<0.05 *$ \\
\hline $\mathrm{a} / \mathrm{v}$ & $0,77 \pm 0,19$ & $0,75 \pm 0,18$ & $>0.05$ \\
\hline
\end{tabular}

Calculation of the stroke volume is according to the formula of Shramek-Bernstein [13].

*Total examined- 68, men-30, women-38, ONAP-optimal, normal arterial pressure, HNAP-high normal arterial pressure

HR- Heart rate; TFC-Thoracic fluid content; VI- Velocity index; SV -Stroke volume; CO- Cardiac output; CI- Cardiac index; SVRI- Systemic vascular resistance index; LCWI-Left cardiac work index ; STR-Systolic time ratio; MBP- Mean blood pressure; $\mathrm{a} / \mathrm{V}$ - mean arterial / venous capillary diameter. 
Fig.1: Crossed capillaries in HNAP (Capillaroscope JH1005, PRC magnification ×250. )

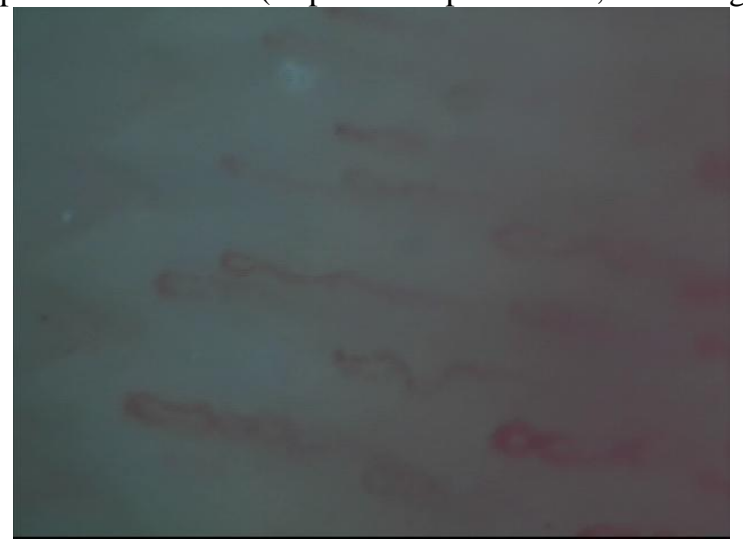

\section{References:}

1. Ezzati M, Lopez AD, Rodgers A et al (2002). Selected major risk factors and global and regional burden of disease. Lancet.,360 (9343):1347-60.

2. Varsan RS, Larson MG, Leip EP et al (2001). Impact of high-normal Blood pressure on the risk of cardiovascular disease. N Engl J Med., 345: 1291-1297.

3. Reappraisal of European guidelines of hypertension management. (2009) J Hypertens., 27(11):2121-2158.

4. Gupta AK, Mc Glone M, Greenway FL et al. (2010). Prehypertension in disease-free adults: a marker for an adverse cardiometabolic risk profile. Hypertens Res., 33: 905-910

5. Lee M, Saver J, Chang B et al. (2011) Presence of baseline prehypertension and risk of incident stroke - a metaanalysis. Neurology., 77: 1330-1.

6. Conroy RM, Pyörälä K, Fitzgerald AP et al.(2003) Estimation of ten-year risk of fatal cardiovascular disease in Europe: the SCORE project. Europ Heart., 24: 987-1003.

7. He FJ, Mac Gregor G.A (2009). A comprehensive review on salt and health and current experience of worldwide salt reduction programmes. J Hum Hypertens, 23(6):363-84.

8. Dickinson HO, Mason JM, Nikolson DJ, Compbell F, Beyer FR, Cook JJ, Williams B, Ford GA.(2006) Lifestyle interventions to reduce raised blood pressure: a systematic review of randomized controlled trials. $J$ Hypertens., 24(2):215-33.

9. Vita JA, Keaney JF. (2002)Endothelial function a barometer for cardiovascular risk? Circulation., 106: 640642.

10. Wang Y, Wang Q J (2004). The prevalence of prehypertension and hypertension among US adults according to the new National Joint Committee Guidelines.Arch Int Med., 164 (19): 2126-34.

11. Gray DS, Fujioka K.(2004) Use of relative weight and Body Mass Index for the determination of adiposity.J Clin Epidemiol., 44 (6): 545-50.

12. Berrington de Gonzalez A, Hartge P, Cerhan JR, Flint AJ, Hannan L, MacInnis RJ et al.(2010) Body-Mass Index and Mortality among 1.46 Million White Adults. N Engl J Med., 363 (23): 2211-9.

13. Biopac MP hardware and software manuals.Biopac systems, inc.

14. Smolle KH, Schmid M, Prettenthaler H, Weger C.(2010) The Accuracy of the CNAP Device Compared with Invasive Radial Artery Measurements for Providing Continuous Noninvasive Arterial Blood Pressure Readings at a Medical Intensive Care Unit: A Method-Comparison Study.Anesth Analg.,121(6):1508-16.

15. Fortin J, Marte W, Grüllenberger R, Hacker A, Habenbacher W, Heller A et al.( 2006) Continuous non-invasive blood pressure monitoring using concentrically interlocking control loops.Computers in biology and medicine., 36(9), 941-57.

16. Ventura HO, Taler SJ, Strobeck JE.(2005) Hypertension as a hemodynamic disease: the role of impedance cardiography in diagnostic, prognostic, and therapeutic decision making. Am J Hypertens.; 18: 26-43.

17. Sanidas EA, Grammatikopoulos K, Anastasiadis G, Papadopoulos D, Daskalaki M, Votteas V(2009). Thoracic fluid content and impedance cardiography: a novel and promising noninvasive method for assessing the hemodynamic effects of diuretics in hypertensive patients. Hellenic J Cardiol., 50(6): 465.

18. Van De Water JM, Miller TW, Vogel RL, Mount BE, Dalton ML.(2003) Impedance cardiography: the next vital sign technology? Chest., 123:2028. 
19. Ilies C, Bauer M, Berg P, Rosenberg J, Hedderich J, Bein B, Hinz J, Hanss R.(2012) Investigation of the agreement of a continuous non-invasive arterial pressure device in comparison with invasive radial artery measurement. Br J Anaest.,; 108(2): 202-10.

20. Shore AC, Tooke JE.(1994) Microvascular function in human essential hypertension. J Hypererens., 12(7): 717-728.

21. Struijker - Boudier HA, le Noble JL, Messing MW et al.(1992) The microcirculation and hypertension . $J$ Hypererens., 10(7): 147-156.

22. Ingegnoli F, Gualtierotti R, Lubatti Ch, De Angelis R.(2013) Nailfold capillary patterns in healthy subjects: A real issue in capillaroscopy.Microvasc. Res., Nov;90:90-5. Abstract.

23. Antonios TF, Singer DR, Markandu ND et al.(1999) Rarefaction of skin capillaries in borderline essential hypertension suggests an early structural abnormality ". J Hypertens., 34: 655-658.

24. Nikolov FP, Nikolov P, Nikolova J. (2011) Prevalence and Risk Profile of Prehypertension in South Bulgaria . International Conference on Prehypertension and Cardio Metabolic Syndrome, 24-27 February, p127.

25. Wang Y, Wang QJ.(2004) The prevalence of prehypertension and hypertension among US adults according to the new National Joint Committee Guidelines. Arch Int Med., 164 (19):2126-34.

26. Greenlund KJ, Croft JB, Mensah GA.(2004) Prevalence of heart disease and stroke risk factors in persons with pre hypertension in the United States. Arch. Int. Med., 164(19):2113-8.

27. Elaine M. Urbina, Philip R. Khoury,Connie McCoy, Stephen R. Daniels, Thomas R. Kimball and Lawrence M. Dolan.(2011) Cardiac and Vascular Consequences of Pre-Hypertension in Youth. J Clin Hypertens., (Greenwich). 13(5): 332-342.

28. O’Rourke M (1995). Mechanical principles in arterial disease. Hypertension; 26(1): 2-9 